\title{
A Multi Functional Plug-in for Exergames
}

\author{
Martina Eckert, Ignacio Gómez-Martinho, Juan Meneses, José F. Martínez Ortega
}

\begin{abstract}
This paper describes the development of a software complement for Blender (a freely available animation software) which allows to insert motion data obtained from a motion capture camera device. The main target is to provide Blender with a tool to develop exergames, i.e. serious games for performing physical exercises, which are fully adaptive to the user's needs and capabilities, especially addressing chronical patients or handicapped. The plugin incorporates both: motion control and recording, allowing to store user movements. Transmission is realized via the OSC (Open Sound Control) protocol. The tool is still under development, here a first version is presented showing its functionalities in a demo-game. In the future, sensor data from other devices is planned to be integrated.
\end{abstract}

\section{INTRODUCTION}

With the upcoming technologies, many new proposals for applications in health, especially in rehabilitation, are arising, which are based on serious gaming in combination with special corporal-sensor devices like handheld pointing devices, balance boards or depth sensing cameras. On one hand it has been proven to be quite effective to perform exercises while playing games and on the other hand the mentioned devices have been tested exhaustively for being valid to measure and reproduce human gestures, e.g. by [1].

Reviewing the myriad of applications already created for different health problems, ages etc., it has been observed that certain lacks are reported frequently, e.g. the software should be adaptable to individual needs, (i.e. in motion range, required speed etc.), easy to configure by the user or an nonexpert assistant; it could be even smart and decide the right exercise every time depending on the user's condition or development, it should be also made for wheelchair users, and above all, the game should be exciting and compelling to motivate the player while doing his exercises without being conscious. This last point is the most important one in our opinion, as exercises usually need a lot of self-discipline, but people who depend on doing them to improve their live conditions like e.g. muscle dystrophy patients, who should not rest but are getting tired very quickly, could benefit a lot on those types of games because they get challenged by them and meanwhile exercise their body.

So the aim of the proposed tool is to cover those lacks by providing a smart connection between the powerful animation software Blender and a motion capture camera. Those devices are cheap and proven to be effective with corporal motion

This work was sponsored by Spanish National Plan for Scientific Tech Res. and Innov.: TEC2013-48453-C2-2-R. detection. As a demonstration, a $3^{\text {rd }}$ person Adventure game is presented to depict the possibilities for implicit exercises and their individual configuration.

\section{SIMILAR APPROACHES}

When literature has been browsed for similar approaches, the only two interfaces between motion capture devices and Blender were found, namely "Blender Loop Station" (Bloop) [2], not further updated, and a commercialized middleware [3] which transmits camera data to Blender or other software. The Bloop codec is public, but not working on new equipment, the other tool has to be purchased and does not cover all our prospects, so the authors preferred not to use any of the before mentioned tools and implement an own open-ended middleware.

Relating the usage of Blender in e-health applications, two proposals have been found, both without motion capture camera: Juhasz et al. [4] created a 3D visualization tool to control the patient's movements by visualizing motion data on a humanoid avatar, and Scardovelli [5], who implemented an adventure game with avatar, controlling its movements with help of image areas and a webcam.

Generally, there are lots of proposals for physical exercises or rehabilitation that make use of a mocap device in combination with other software than Blender. The one presented in [6] is especially interesting for comparison with the here presented, as it also focuses on wheelchair users. The software offered in [7] is also interesting, but all have one lack: they provide very typical exercises, e.g. to move some toy, to shoot balloons or to cross labyrinths. What is missed, is an exciting "storyboard" that involves the necessary movements into a challenging environment and mixes up different exercises to avoid boredom and repetitive procedures.

\section{SYSTEM PROPOSAL}

\section{A. Data provider and Blender Addon}

Only one freely available and working tool was found in [8], which captures images and calculates skeleton data, providing it afterwards to another application. It has been implemented based on SDK library functions provided with the camera. The skeleton data is coded accordingly to the OSC (Open Sound Control) protocol and then sent to any configurable port.

As a starting point, the proposed Blender add-on has been developed based on the output of the mentioned tool, currently, an own middleware is under development. The free 
PythonOSC [9] library has been used to read the chains of text and floats received from a configurable port. Concretely, the names of the joints and their projective coordinates $\mathrm{X}, \mathrm{Y}$ (image plane) and $Z$ (distance to the camera) are obtained. See the aspect of the Blender plug-in in Fig. 1.
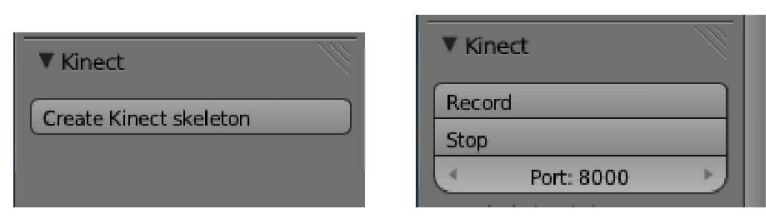

Fig. 1 Blender add-on, pre-operative state (left), operative state (right).

\section{B. Third person adventure demo-game}

To perform preliminary tests with the add-on, a short adventure game has been implemented (Fig. 2). It constitutes a third person game with an avatar seen from backwards, which represents the player. In two scenes, different obstacles have to be conquered to prove different functionalities like jumping, bending down, reaching things and cutting barriers. It combines motion control with predefined animations, realized the recording function of the add-on.

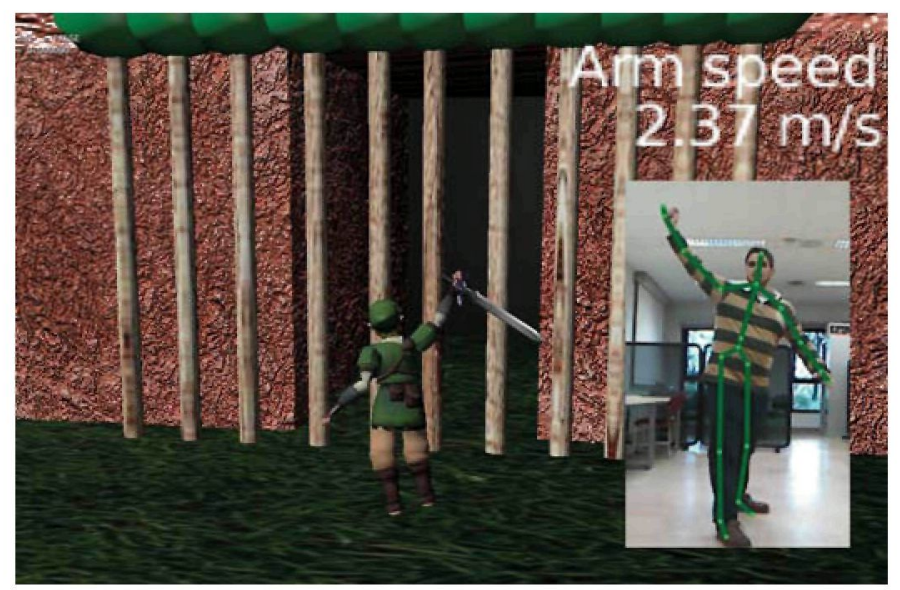

Fig. 2 Typical scene of the demo-game with user overlaid.

The game itself is not the focus of the presented work, but the functionalities provided through the plugin:

1. Different avatars: The plugin provides an invisible skeleton used to copy the movements of the corresponding articulations to any desired avatar.

2. Seated mode: Upper body joints are assigned to the real movements while lower joints are animated for walking.

3. Mobility: While the user is not advancing in front of the camera, the avatar has to get around in a huge virtual world. Here, real and animated movements have been combined and restrictions concerning the obstacles existing in the virtual world have been defined.

4. Differentiation of movements: Some are commands to move the avatar (jumping, running, or kneel down), others are defined as game-commands (open the menu) and other are simply copied (reach for an object) or copied.

5. Record user movements. Some animations like walking have been recorded and integrated into the game. This option offers huge possibilities, e.g. the user's movements could be shown to improve, to repeat a success (in slow motion) or to include own gestures into the game etc.

\section{CONCLUSIONS AND FUTURE WORK}

A simple plugin with promising capacities is presented which builds the basis for the creation of user adaptive, easy to configure and exciting exergames for rehabilitation and daily physical exercises in Blender, above all useful for chronical patients which need to do them without being conscious.

The work on the tool is still in process and the following enhancements are actually under development:

\section{a) Configuration of user specific parameters}

If the user has a limited motion range, cannot move fast or often, the avatar will be ordered to enhance the movements such that the user shouldn't notice it. In the same way, the tool would measure the fatigue and react in a smart way: slower and less powerful movements invoke the avatar to compensate them and adapt the difficulty of the exercises. The enhancement of restricted movements can be a psychological motivation to do things, the user in real life couldn't do.

\section{b) Wheelchair mode}

Although the user needs a wheelchair, the avatar would be walking and running through an animation. The player would perform upper body exercises and control the direction and speed of the avatar.

\section{c) Further sensor data}

Other devices like mobile phone or heart rate control could be incorporated with the idea to give way for a smart context aware software that fully adapts the exercises to the user's necessities.

\section{REFERENCES}

[1] M. A. Fraiwan, N. Khasawneh, A.Malkawi, M. Al-Jarrah, R. Alsa'di, and S. Al-Momani, "Therapy central: On the development of computer games for physiotherapy", 9th Int. Conf. on Innovations in Information Technology (IIT), 24-29, 2013.

[2] F. Biermann, N. Steenbergen and B. Walther-Franks, „Blender Loop Station (Bloop)", online: http://dm.tzi.de/bloop/ last update: 1 Oct. 2012, [visited: 30 Jan. 2015]

[3] Delicode Ltd., "NI Mate and Blender", online: http://www.nimate.com/use/blender/, [visited: 16 March 2015].

[4] N. Juhasz, B. Juhasz, H. Steiner, and Z. Kertesz, "Biomechanical equipment development in Virtual Collaboration Arena," IEEE 4th Int. Conf. on Cognitive Infocommunications, pp. 447-452, 2013.

[5] T. A. Scardovelli and A. F. Frere, "The design and evaluation of a peripheral device for use with a computer game intended for children with motor disabilities," Computer Methods and Programs in Biomedicine, vol. 118, pp. 44-58, Jan. 2015.

[6] R. N. Madeira, L. Costa, and O. Postolache, "PhysioMate - Pervasive physical rehabilitation based on NUI and gamification," Int. Conf. and Exposition on Electrical and Power Engineering, pp. 612-616, 2014.

[7] Brontes Processing (Ltd.), "SeeMe", online: http://www.virtual-realityrehabilitation.com/products/seeme/what-is-seeme [visited: 23 March 2015].

[8] A. Hayter, "KinectOSC - A utility to transmit data from official Microsoft Kinect SDK via OSC", online: https:/github.com/ahsquared/ KinectOSC last update 22 Oct. 2013, [visited 16 March 2015].

[9] Python Software Foundation, "Pyhton-OSC library", online: https:/pypi.python.org/pypi/python-osc, last update: 36 Oct. 2014, [visited: 30 Jan. 2015]. 